\title{
Infrared modulated thermography based on the adaptive complex Morlet wavelet analysis
}

\author{
by Xingwang Guo*, Shiqi Zhao* and Yingtao Liu**
}

* Beihang University, School of Mechanical Engineering and Automation, 37 Xueyuan Rd. Haidian District, 100191, Beijing, China, xingwangguo@buaa.edu.cn yingt.liu@163.com

${ }^{\star *}$ AECC Beijing Institute of Aeronautical Materials, Huanshangcun. Haidian District, 100095, Beijing, China,

\section{Abstract}

An adaptive infrared modulated image sequence processing method based on the complex Morlet wavelet analysis is proposed for the modulated thermography of composite materials, focusing on the complicated parameter selections. A wavelet scale selection algorithm is proposed according to the modulation frequency of thermal excitation and the sampling frequency of infrared imaging. The phase images obtained using this method and FFT are compared. The results show that the method can select the scale parameter of wavelet analysis automatically, and has a good tolerance to the change of modulation frequency.

\section{Introduction}

Active infrared non-destructive testing (IR NDT) is one of the widely used NDT techniques [1]. IR NDT is usually carried out by exciting specimens actively. In terms of different thermal excitation forms, IR NDT can be classified into pulsed thermography (PT), modulated thermograpy (MT), stepped thermography (ST) [2], frequency modulated thermal wave imaging (FMTWI) [3], etc.. MT, also known as lock-in thermography (LT), is one of the most widely used schemes in the defect detection of composite materials, due to its advantages of low power heating source, good tolerance for uneven heating and surface conditions. In MT, halogen lamps are usually used to stimulate the inspected specimen at a selected modulated frequency. Since the internal structure and thermal performance of defect areas and non-defect areas are different, the amplitude and phase of thermal signal are also different in the two areas during the thermal wave propagation. By analyzing the surface thermal wave signals to obtain the phase or amplitude difference, internal defects can be detected and identified. At present, the fast Fourier transform (FFT) is the commonly used signal processing method for MT, and the wavelet transform (WT) can also be used successfully [4]. The results in literature [4] by Robert Olbrych showed that the processing result obtained by the complex Morlet WT was similar to that of the FFT, and could characterize the transient process of the infrared modulated signal.

In the complex Morlet wavelet analysis, the selection of wavelet transform scale parameter plays a very important role. However, the scale parameter selection is usually determined by human experience with trial-and-error procedure and huge calculation. Therefore, it is of great significance to seek an adaptive scale selection algorithm for the complex Morlet wavelet analysis. In this paper, the infrared modulated image sequence processing method based on the complex Morlet WT is proposed focusing on the automatic selection of the scale factor.

\section{Complex Morlet wavelet transform}

The continuous wavelet transform (WT) is defined as Eq.(1) [5].

$$
W T(a, \tau)=\int_{R} x(t) \psi_{s, \tau}^{*}(t) d t
$$

where $x(t)$ is the analyzed signal, $\psi_{s, \tau}(t)$ is a set of basis function as called wavelets, * stands for complex conjugation, $W T(a, t)$ is the wavelet transform coefficients. $\psi_{s, \tau}(t)$ is translated from the parent wavelet $\psi(t)$ by scale (or frequency) factor $s$ and time factor $\tau$, defined as Eq.(2).

$$
\psi_{s, \tau}=\frac{1}{\sqrt{a}} \psi\left(\frac{t-\tau}{a}\right)
$$

$W T(a, \tau)$ is decomposed into the real part $W T_{\mathrm{R}}(a, \tau)$ and the imaginary part $W T_{1}(a, \tau)$, and the amplitude $A(a, \tau)$ and phase $\varphi(a, \tau)$ of $W T(a, \tau)$ are defined by Eq.(3) and Eq.(4)

$$
A(a, \tau)=\sqrt{W T_{\mathrm{R}}(a, \tau)^{2}+W T_{1}(a, \tau)^{2}}
$$




$$
\varphi(a, \tau)=\left\{\begin{array}{cc}
\arctan \left(W T_{1}(a, \tau) / W T_{\mathrm{R}}(a, \tau)\right) & W T_{\mathrm{R}}(a, \tau)>0 \\
\arctan \left(W T_{1}(a, \tau) / W T_{\mathrm{R}}(a, \tau)\right)+\pi & W T_{\mathrm{R}}(a, \tau)<0, W T_{1}(a, \tau) \geq 0 \\
\arctan \left(W T_{1}(a, \tau) / W T_{\mathrm{R}}(a, \tau)\right)-\pi & W T_{\mathrm{R}}(a, \tau)<0, W T_{1}(a, \tau)<0 \\
\frac{\pi}{2} & W T_{\mathrm{R}}(a, \tau)=0, W T_{1}(a, \tau)>0 \\
0 & W T_{\mathrm{R}}(a, \tau)=0, W T_{1}(a, \tau)=0 \\
-\frac{\pi}{2} & W T_{\mathrm{R}}(a, \tau)=0, W T_{1}(a, \tau)<0
\end{array}\right.
$$

According to the reference [4], the complex Morlet wavelet is chose as the parent wavelet. The function of the complex Morlet wavelet in time domain is defined as Eq.(5).

$$
\psi(t)=\frac{1}{\sqrt{\pi f_{\mathrm{b}}}} \exp \left(-\frac{t^{2}}{f_{\mathrm{b}}}+j 2 \pi f_{\mathrm{c}} t\right)
$$

where $f_{\mathrm{b}}$ is the bandwidth of the complex Morlet wavelet, and $f_{\mathrm{c}}$ is the center frequency of the complex Morlet wavelet. Take $f_{\mathrm{b}}=1 \mathrm{~Hz}, f_{\mathrm{c}}=1 \mathrm{~Hz}$.

\section{Experimental setup and sample}

The experimental set up is a self-developed modulated thermography system which consists of an uncooled FPA IR camera, double halogen lamps, a thermal excitation controller, a computer, and the specific software, as shown schematically in Fig.1. The resolution of the IR camera is 320 pixel $\times 240$ pixel, the temperature sensitivity is $0.08 \mathrm{~K}$ and the maximum frame rate is $60 \mathrm{~Hz}$. The double halogen lamps with the total power of $2600 \mathrm{~W}$ illuminate the sample with $45^{\circ}$ incidence angle, and the distance from the lamps to the sample center is $45 \mathrm{~cm}$. The IR camera was placed in front of the sample at a distance of $64 \mathrm{~cm}$, and at the same side with the lamps. Parameters of thermal excitation and image acquisition are set in the application software so that the lamps emit a harmonic heat wave according to the given modulation frequency, and the IR camera synchronously records the change of the surface temperature of the sample.

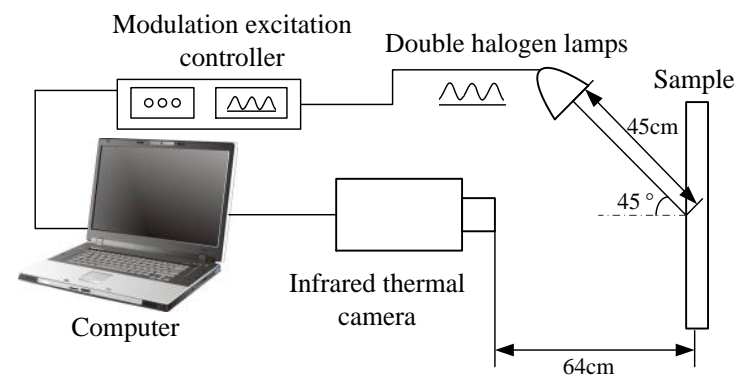

Fig.1. Scheme of the modulated thermography system

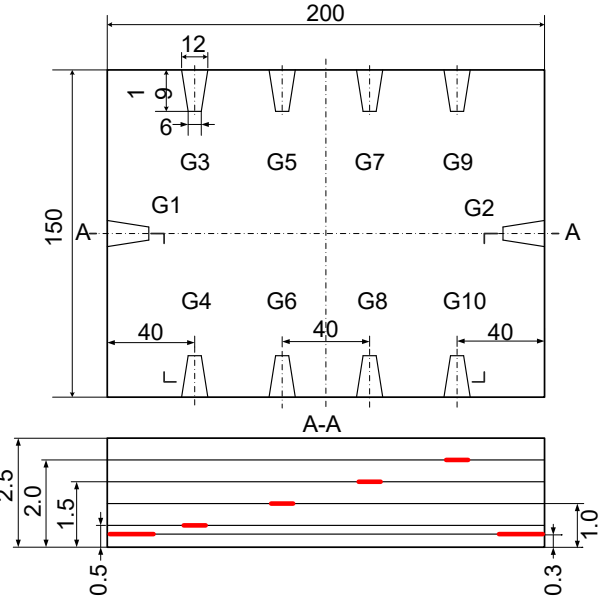

Fig. 2. Schematic diagram of the GFRP sample

The samples is a glass fiber reinforced plastic (GFRP) plate, as shown in Fig. 2. It has internal delamination defects at different depths. The defects were made by inserting into a stainless steel sheet with a thickness of $0.05 \mathrm{~mm}$, and then pulling it out after forming. The shape of the steel sheet is a trapezium with the bottom of $12 \mathrm{~mm}$, the top of 6 $\mathrm{mm}$, the height of $19 \mathrm{~mm}$ and the area of $171 \mathrm{~mm}^{2}$. In order to increase the heat absorption and emissivity on the tested surface, the tested surface of the GFRP sample are coated with black paint.

The dimension of the GFRP sample is $200 \mathrm{~mm} \times 150 \mathrm{~mm} \times 2.5 \mathrm{~mm}$. There are 5 pairs of delamination defects embedded at 5 different depths along the side edges of the sample. The depths of the five pairs of defects are $0.3 \mathrm{~mm}$, $0.5 \mathrm{~mm}, 1.0 \mathrm{~mm}, 1.5 \mathrm{~mm}$ and $2.0 \mathrm{~mm}$ respectively. 


\section{The adaptive complex Morlet wavelet analysis of the modulated thermal signal}

\subsection{Effective scale}

In wavelet analysis, the scale factor $a$ is relative to frequency domain resolution. At the scale $a$, the center frequency $f_{a}$ of the wavelet is defined as Eq.(9):

$$
f_{a}=f_{\mathrm{c}} \cdot f_{\mathrm{s}} / a
$$

where $f_{\mathrm{s}}$ is the sampling frequency, $f_{\mathrm{c}}$ is the center frequency of the parent wavelet. When the frequency of analyzed signal is closer to $f_{a}$, the values of the wavelet transform coefficients are higher, and could characterize the analyzed signal better.

At time $t$, if the modulus of wavelet transform coefficients is maximum at the scale $a$, then the local frequency $f$ of the analyzed signal at time $t$ is similar to or the same as the center frequency $f_{a}$ of the wavelet at the scale $a$. So at the specific scale $a$, which is defined as the effective scale $a_{\mathrm{e}}$, the analyzed signal can be highly characterized. In other words, at the scale $a_{\mathrm{e}}$, the analyzed signal can be effectively characterized by wavelet transform coefficients.

\subsection{Wavelet scale selection}

The actual modulated wave signal is a superposition of a single frequency cosine signal, a transient response signal, low frequency noise and high frequency noise. By using the multi-scale characteristics of the wavelet transform and considering the modulation frequency of the thermal excitation, the cosine signal could be extracted from the actual modulated wave signal. When the modulation frequency is closest to one wavelet basis's center frequency among the others, the wavelet coefficients of this wavelet basis could effectively represent the cosine signal component. So the scale, which is corresponding to this wavelet basis, is an effective scale $a_{\mathrm{e}}$ of the modulated wave signal. In order to find $a_{\mathrm{e}}$ quickly, the center frequency of each wavelet bases could be equally spaced in the frequency domain. Defining decomposition level of wavelet transformation as $M$, the $i$-th scale as $a_{i}$, and the center frequency of the wavelet basis at the scale $a_{i}$ as $f_{a i}$, then let $f_{a i}$ satisfy the Eq.(10).

$$
f_{a i}=f_{\mathrm{s}} \cdot(M+1-i) / 2 M \quad i=1,2, \cdots, M
$$

According to the Eq.(9), $f_{a i}$ could be obtained by Eq.(11).

$$
f_{a i}=f_{\mathrm{c}} \cdot f_{\mathrm{s}} / a_{i}
$$

Based on Eq.(10) and Eq.(11). $a_{i}$ is calculated as Eq.(12).

$$
a_{i}=2 f_{\mathrm{c}} M /(M+1-i) \quad i=1,2, \cdots, M
$$

Eq.(11) and Eq.(12) show that when $f_{a i}$ is equally spaced in the frequency domain, $f_{a i}$ is related to the decomposition level $M$ and sampling frequency $f_{\mathrm{s}}$, but is unrelated to the bandwidth $f_{\mathrm{b}}$ and the center frequency $f_{\mathrm{c}}$ of the parent wavelet.

In order to figure out the noise distribution in the actual modulated wave signal, two sets of signal were adopted. One was an actual modulated wave signal. The other was an ideal stable wave signal at a single frequency. The actual modulated thermal wave signal, named as $\theta_{1}$, was acquired by the IR camera with sampling frequency $f_{\mathrm{s}}=1 \mathrm{~Hz}$. The modulation frequency $f$ of the excitation source was $0.0625 \mathrm{~Hz}$. The IR camera recorded an image sequence in three modulation periods. The sampling number was $N=48$. The ideal stable wave signal was named as $\theta_{2}$. Its expression was shown as Eq.(13).

$$
\theta_{2}(n)=1+\sin \left(2 \pi f \frac{n}{f_{\mathrm{s}}}-\frac{\pi}{2}\right), \quad n=0,1, \cdots, N-1
$$

where $f=0.0625 \mathrm{~Hz}, f_{\mathrm{s}}=1 \mathrm{~Hz}$, and $N=48$.

Take four sets of decomposition level $M$ to explore the effective scale $a_{\mathrm{e}}$ for the signals $\theta_{1}$ and $\theta_{2}$. The decomposition level $M$ of the wavelet analysis was selected as shown in Table 1. After calculation, the time-frequency diagrams of both signals were shown in Fig. 3 and Fig.4 respectively. 
Table1. The choice of the decomposition level M

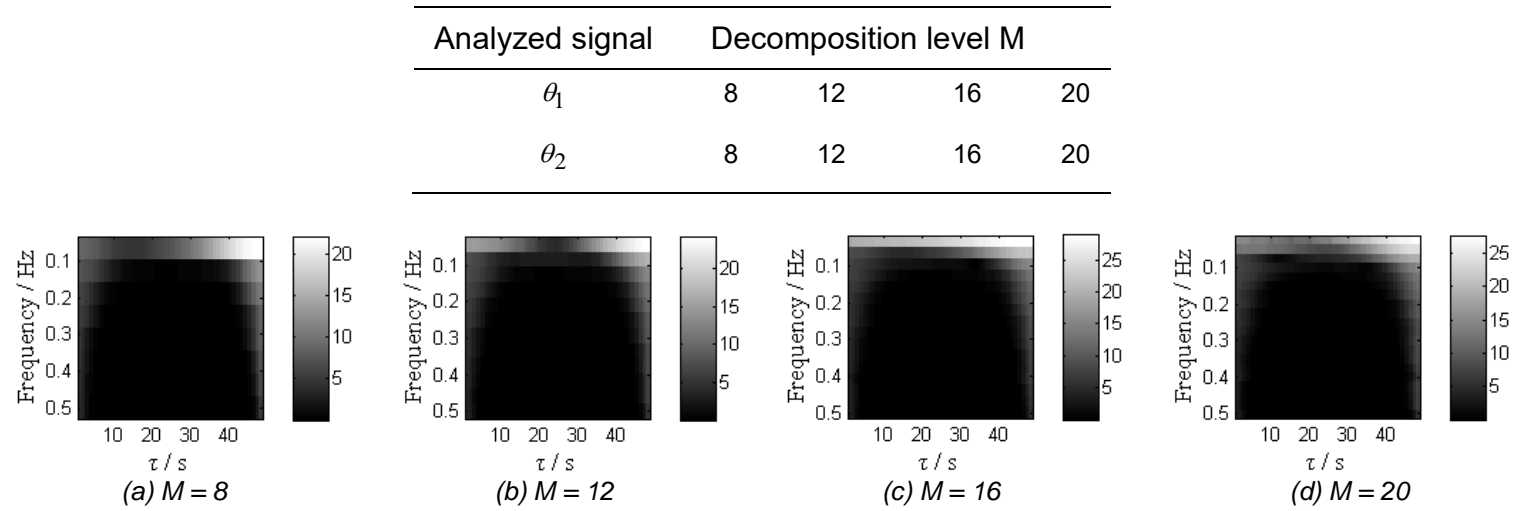

Fig.3. The time-frequency diagrams of the wavelet transform coefficients of signal $\theta_{1}$

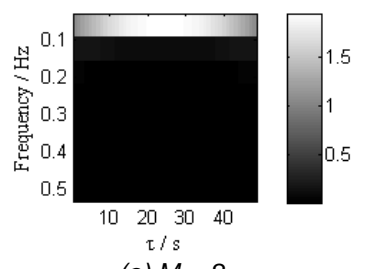

(a) $M=8$

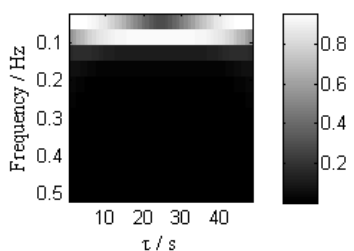

(b) $M=12$

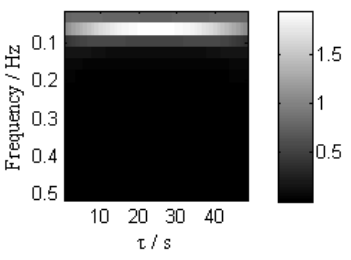

(c) $M=16$

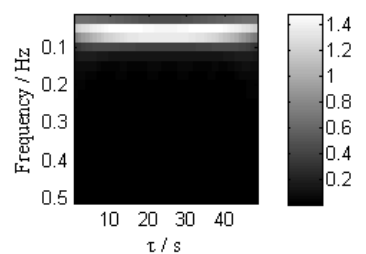

(d) $M=20$

Fig.4. The time-frequency diagrams of the wavelet transform coefficients of signal $\theta_{2}$

The results show that the actual modulated thermal wave signal contains much low-frequency noise in contrast with the ideal stable wave signal. Besides, when $M$ was too small, the frequency resolution of wavelet analysis became so low that low-frequency noise and the cosine signal component couldn't be distinguished. Hence, a suitable decomposition level $M$ could make the modulation frequency $f$ of the cosine signal component lie in the frequency range of the scale $a_{M-1}$, so as to effectively characterize the modulated wave signal and reduce low- frequency and high-frequency noise. Since the center frequency of each wavelet base was equally spaced, so $f$ could be easily satisfied as Eq.(14).

$$
\frac{f_{a(M-1)}+f_{a M}}{2} \leq f \leq \frac{f_{a(M-1)}+f_{a(M-2)}}{2}
$$

where $f_{a M} 、 f_{a(M-1)} 、 f_{a(M-2)}$ are the center frequencies of the wavelet bases at the scale $a_{M} 、 a_{M-1} 、 a_{M-2}$. Based on Eq.(10) and Eq.(14), the decomposition level $M$ could be determined by Eq.(15).

$$
3 f_{\mathrm{s}} /(4 f) \leq M \leq 5 f_{\mathrm{s}} /(4 f)
$$

When $M$ satisfied Eq.(15), the wavelet transform coefficients at the scale $a_{M-1}$ could represent the cosine signal component of the actual modulated wave signal. In other words, the scale $a_{M-1}$ is just the effective scale $a_{\mathrm{e}}$ for the modulated wave signal, i.e. $a_{\mathrm{e}}=a_{M-1}$. In order to increase the accuracy, let $M$ take the right value of Eq.(15). Therefore, the formula for $M$ is defined as Eq.(16).

$$
M=5 f_{\mathrm{s}} /(4 f)
$$

\subsection{Phase image sequence of wavelet transform coefficients}

According to Eq.(4), the phase of the WT coefficients would be abruptly changed in calculation, causing a lot of noise in the phase image at some moment. For example, set the modulation frequency $f=0.0625 \mathrm{~Hz}$, a thermal image sequence was acquired by the IR camera with sampling frequency $f_{\mathrm{s}}=1 \mathrm{~Hz}$, sampling time $T=48$ s and resolution 320 pixel $\times 240$ pixel. According to Eq.(16), the decomposition level is $M=20$, then the effective scale $a_{\mathrm{e}}=a_{19}$. The temperature curve of the modulated thermal wave signal at a typical pixel is presented in Fig. 5 , and the corresponding 
phase curve of the wavelet coefficient at the effective scale of the signal is shown in Fig. 6 . It can be seen that the period of the wavelet coefficient phase curve is the same as the modulation period of the modulated thermal wave signal. Fig.7 is one of phase images of the wavelet transform coefficients when the phases are in the continuity interval, and Fig. 8 is one of phase images of the wavelet coefficients when the phases are at the discontinuity points.

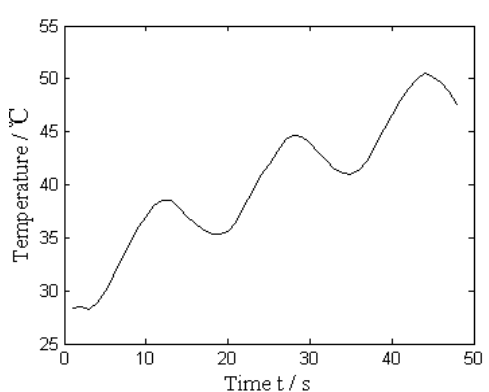

Fig.5. A typical curve of the modulated thermal signal

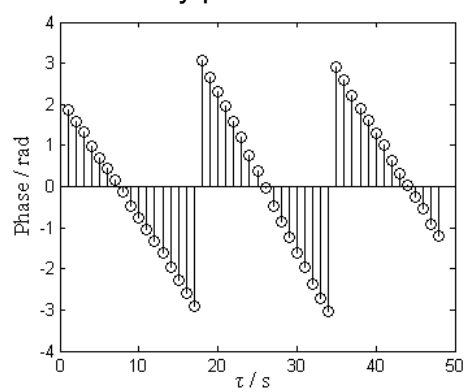

Fig.6. The corresponding phase curve of the wavelet coefficients with the effective scale

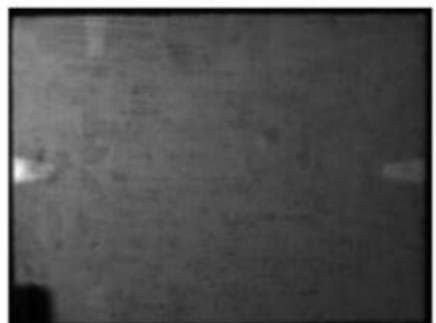

(a) $\tau=5 \mathrm{~s}$

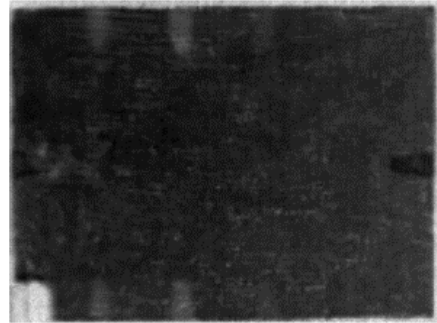

(b) $\tau=30 \mathrm{~s}$

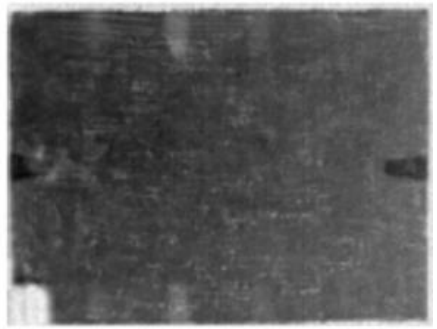

(c) $\tau=40 \mathrm{~s}$

Fig.7. Phase images of the wavelet transform coefficients when the phases are in the continuity interval

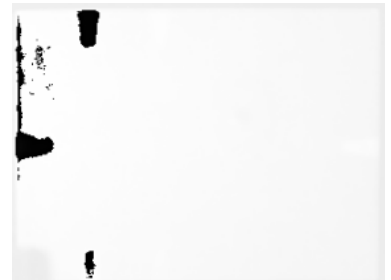

(a) $\tau=18 \mathrm{~s}$

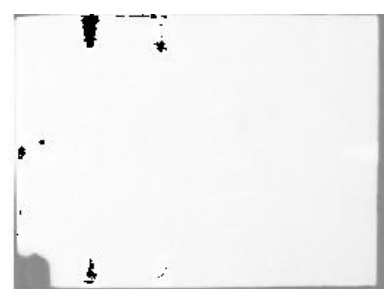

(b) $\tau=37 \mathrm{~s}$

Fig.8. Phase images of the wavelet transform coefficients when the phases are at the discontinuity points

Due to the internal inconsistency, the thermal wave is transmitted unevenly inside the specimen. As seen from the two sets of images in Fig.7 and Fig.8, the phase singular values at the discontinuity points result in excessive noise in the phase image and badly affect the identification of defects. According to Fig.5 and Fig.6, the period of the wavelet coefficient phase is consistent with the modulation period of the thermal excitation. Therefore, the thermal excitation modulation period could be used to locate and remove the noisy images which are the phase images of the wavelet coefficients at the start of each modulation period, the end of each modulation period and their neighborhoods.

\subsection{Steps of thermal image sequence processing using the adaptive complex Morlet WT}

According to the above, $M$ and $a_{e}$ can be selected adaptively based on the modulation frequency of thermal excitation and the sampling frequency of infrared imaging. The steps for processing the modulated thermal image sequence by using the adaptive complex Morlet WT can be summarized as follows:

(1) Getting $M$ by Eq.(16) and taking the effective scale $a_{\mathrm{e}}=a_{M-1}$;

(2) Performing the complex Morlet WTof the signals at each pixel of the thermal image sequence;

(3) Extracting phase images of the wavelet transform coefficients at the effective scale $a_{\mathrm{e}}$, and removing phase images at the starts and ends of the modulation periods. 


\section{Experimental result}

The thermal image sequences acquired by the modulated thermography are processed by the complex Morlet WT and the FFT respectively. In order to evaluate advantages and disadvantages of both methods in characterizing the defects at different depths, the signal-to-noise ratio (SNR) is selected as the quantitative evaluation criteria of the processing effects. The SNR is defined as Eq.17,

$$
S N R=\left|\bar{T}_{\mathrm{d}}-\bar{T}_{\text {nd }}\right| / \sigma_{\text {nd }}
$$

where $\bar{T}_{\mathrm{d}}$ and $\bar{T}_{\mathrm{nd}}$ are the average of the phase values in the defect area and non-defect area respectively; $\sigma_{\text {nd }}$ is the standard deviation of the phase values in the non-defect area. The SNR for the adaptive complex Morlet wavelet analysis method is the maximum SNR among the phase sequence of the wavelet coefficients, and the SNR for the Fourier transform phase method is the SNR of the phase image calculated by FTT at the analysis frequency that is equal to, or closest to the modulation frequency.

The GFRP sample was tested at three modulation frequencies, $f=0.0625 \mathrm{~Hz}, f=0.0413 \mathrm{~Hz}$, and $f$ $=0.03125 \mathrm{~Hz}$ respectively. The sampling frequency was $f_{\mathrm{s}}=1 \mathrm{~Hz}$. According to Eq.16, the decomposition level $M$ and effective scale $a_{\mathrm{e}}$ were chosen as Table 2 .

Table 2. $M$ and $a_{\mathrm{e}}$ of the experiments with the GFRP sample

\begin{tabular}{cccc}
\hline No. & Modulation frequency $f(\mathrm{~Hz})$ & decomposition level $M$ & Effective scale $a_{\mathrm{e}}$ \\
\hline 1 & 0.0625 & 20 & 19 \\
2 & 0.0413 & 30 & 29 \\
3 & 0.03125 & 40 & 39 \\
\hline
\end{tabular}

The processing results are shown in Fig.9-Fig.11. Among them, Fig.9(a), Fig.10(a) and Fig.11(a) are the results of the adaptive complex Morlet wavelet analysis method, Fig.9(b), Fig.10(b) and Fig.11(b) are the results of the Fourier transform phase method. Table 3, Table 4 and Table 5 are the SNR of the processing results. Fig.12(a) and Fig.12(b) show the SNR curves obtained by the two methods.

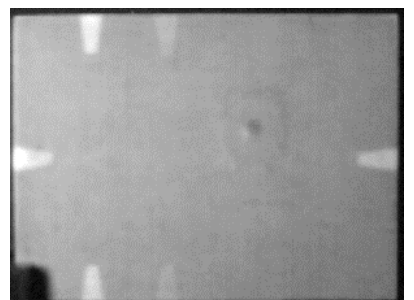

(a)

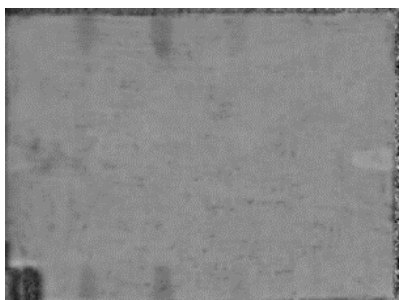

(b)

Fig.9. Experimental results for modulation frequency $f=0.0625 \mathrm{~Hz}$

(a) the phase image of wavelet coefficients with $\tau=24 \mathrm{~s}$, (b) the phase image of FFT method at $f_{0}=0.0625 \mathrm{~Hz}$

Table 3. SNRs of the phase images of the two methods for the modulation frequency $f=0.0625 \mathrm{~Hz}$

\begin{tabular}{cccc}
\hline Defect code & Defect depth $(\mathrm{mm})$ & SNR of WT method & SNR of FFT method \\
\hline G1 & 0.3 & 32.87 & 1.03 \\
G2 & 0.3 & 12.15 & 3.42 \\
G3 & 0.5 & 9.89 & 5.69 \\
G4 & 0.5 & 14.25 & 4.94 \\
G5 & 1.0 & 4.83 & 5.69 \\
G6 & 1.0 & 4.28 & 7.97 \\
G7 & 1.5 & 2.68 & 1.32 \\
G8 & 1.5 & 0.78 & 1.81 \\
\hline
\end{tabular}




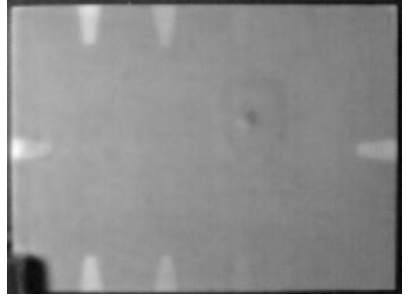

(a)

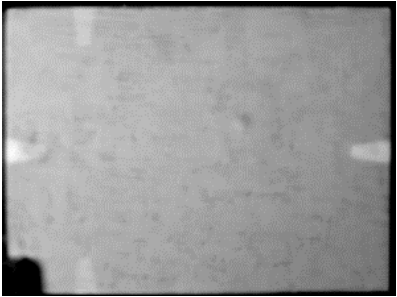

(b)

Fig.10. Experimental results for modulation frequency $f=0.0413 \mathrm{~Hz}$

(a) the phase image of wavelet coefficients with $\tau=69 \mathrm{~s}$, (b) the phase image of FFT method at $f_{0}=0.0430 \mathrm{~Hz}$

Table 4. SNRs of the phase images of the two methods for the modulation frequency $f=0.0413 \mathrm{~Hz}$

\begin{tabular}{cccc}
\hline Defect code & Defect depth $(\mathrm{mm})$ & SNR of WT method & SNR of FFT method \\
\hline G1 & 0.3 & 32.69 & 16.33 \\
G2 & 0.3 & 14.00 & 9.75 \\
G3 & 0.5 & 10.50 & 2.75 \\
G4 & 0.5 & 19.53 & 5.13 \\
G5 & 1.0 & 7.99 & 1.34 \\
G6 & 1.0 & 10.88 & 2.29 \\
G7 & 1.5 & 1.21 & 2.22 \\
G8 & 1.5 & 3.58 & 0.46 \\
\hline
\end{tabular}

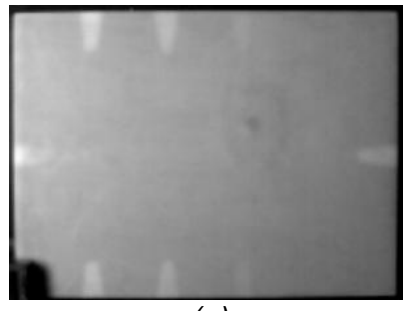

(a)

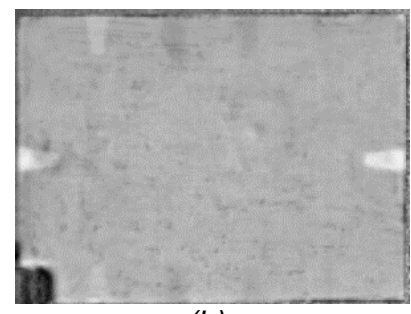

(b)

Fig.11. Experimental results for modulation frequency $f=0.03125 \mathrm{~Hz}$

(a) the phase image of wavelet coefficients with $\tau=51 \mathrm{~s}$, (b) the phase image of FFT method at $f_{0}=0.03125 \mathrm{~Hz}$

Table 5. SNRs of the phase images of the two methods for the modulation frequency $f=0.03125 \mathrm{~Hz}$

\begin{tabular}{cccc}
\hline Defect code & Defect depth $(\mathrm{mm})$ & SNR of WT method & SNR of FFT method \\
\hline G1 & 0.3 & 16.76 & 14.45 \\
G2 & 0.3 & 5.97 & 16.11 \\
G3 & 0.5 & 4.55 & 4.46 \\
G4 & 0.5 & 8.33 & 2.41 \\
G5 & 1.0 & 5.37 & 2.42 \\
G6 & 1.0 & 6.58 & 5.93 \\
G7 & 1.5 & 1.24 & 1.66 \\
G8 & 1.5 & 3.55 & 2.77 \\
\hline
\end{tabular}

For the complex Morlet wavelet phase method, the phase images of wavelet coefficients are not affected by the material surface noise signal. Defect areas in the phase image have clear and complete outline. Regardless of the depth of defects, the defect areas are bright spots in phase image of wavelet coefficients, which means the phase at the defect area always leads the phase at the non-defect area. As the thermal excitation modulation frequency decreases, the deeper defects can be detected. 


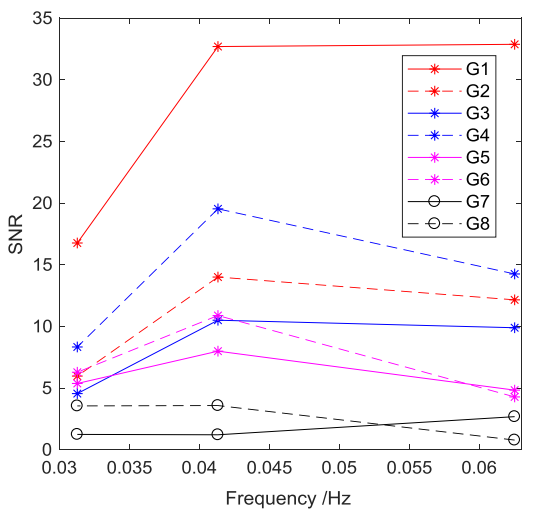

(a)

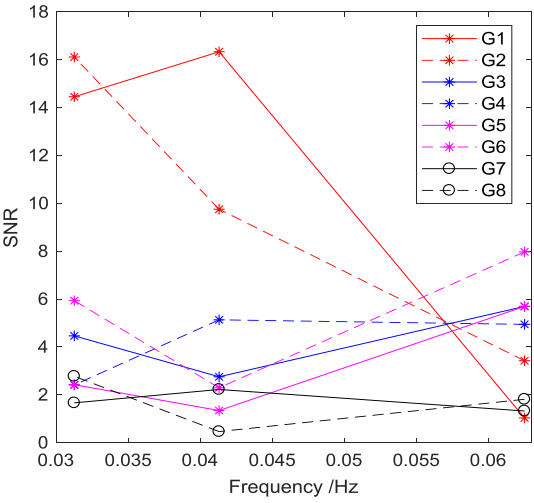

(b)

Fig 12. SNR curves of the GFRP sample

(a) complex Morlet wavelet transform method, (b) FFT method

For the FFT phase method, firstly, when the analysis frequency is equal to the modulation frequency, delamination defects with a depth no greater than $1.5 \mathrm{~mm}$ can be detected, as shown in Figure 9 (b) and Figure 11 (b); when the analysis frequency is not equal to the modulation frequency, the defects at a certain depth may not be detected as shown in Figure 10 (b). Secondly, in Fig.9(b) and Fig.11(b), the shallow defects (e.g. at the depth of $0.3 \mathrm{~mm}$ ) are bright spots, whereas the deep defects are dark spots, that is, the leading and lagging relations between the phase at the defect area and the non-defect area are inversed as the depth of the defect changes. Therefore, the defects at a certain depth must be missed. Finally, the defect outlines of dark spots are blurred. The FFT phase image is greatly affected by the speckles on the sample's surface and contains more noise

\section{Conclusions}

The adaptive wavelet scale selection algorithm for the complex Morlet wavelet analysis is proved effective to extract the effective scale, in which the modulation frequency is.

The adaptive complex Morlet wavelet phase analysis can automatically select the scale parameters (the effective scale and the corresponding decomposition level) of the wavelet analysis and has a good tolerance to the change of the modulation frequency.

The complex Morlet WT phase method can extract defects at different depths steadily. When the defects at different depths exist, the signs of the phase differences between the defect areas and the non-defect area are consistent. Therefore, the complex Morlet WT phase method does not have the situation of missing detect. However, the FFT phase method can obtain a good detection of the defect only at a specific depth corresponding to the modulation frequency, and may cause the missing detection of defects at other depths.

The complex Morlet WT method can remove the low-frequency and high-frequency noise of the modulated thermal imaging signal, and has the capability of extracting the sinusoidal signal at the modulation frequency from the noisy unsteady thermal wave signal.

\section{Acknowledgment}

This work was supported by the National Natural Science Foundation of China (61571028, U1433122).

\section{REFERENCES}

[1] Vavilov V P, Burleigh D D. Review of pulsed thermal NDT: physical principles, theory and data processing. NDT \& E International, 2015; Vol.73, pp.28-52.

[2] Almond D P, Angioni S L, Pickering S G. Long pulse excitation thermographic non-destructive evaluation. NDT\&E International, 2017; Vol.87, pp.7-14.

[3] Ghali V S, Mulaveesala R, Takei M, Frequency-modulated thermal wave imaging for non-destructive testing of carbon fiber-reinforced plastic materials, Meas. Sci. Technol. 2011; Vol.22, No.10, pp.1616-1630.

[4] Robert Olbrycht, Bogusław Więcek, Grzegorz Gralewicz, Tomasz Swiątczak, Grzegorz Owczarek. Comparison of Fourier and wavelet analyses for defect detection in lock-in and pulse phase thermography. Quantitative Infrared Thermography Journal. 2007; Vol.4, No. 2, pp.219-232.

[5] Rioul O, Vetterli M. Wavelets and signal processing. IEEE Signal Processing Magazine. 1991; Vol.8, No.4, pp.14-38. 